\title{
Determination of upper extremity muscle strength profile of Turkish weightlifting national team athletes
}

\author{
Tuğba KOCAHAN ${ }^{1}$, Bihter AKINOĞLU
}

${ }^{1}$ Department of Health Services, Center of Athlete Training and Health Research, Sports General Directorship, The Ministry of Youth and
Sports, Ankara, Turkey.
2 Department of Physiotherapy and Rehabilitation, Faculty of Health Sciences, Ankara Yıldırım Beyazıt University, Ankara, Turkey.

\section{SUMMARY}

This study aimed to determine the upper extremity isokinetic muscle strength profile of Turkey male weightlifters and shed light on the exercise and training program by sharing these results with the athletes and all team working presently in the weightlifting sports area.

This study included 21 weightlifters, who did not have any orthopedic problems, did professional weightlifting for at least 2 years, were cooperative, had a cognitive state required for the assessment, and volunteered to participate in the study. The tests were performed using an isokinetic dynamometer system at angular velocities of $60 \%$ s and $240 \% \mathrm{~s}$ during concentric contractions. The protocol was applied separately to the right and left extremities for the shoulder internal rotation/external rotation and elbow flexion/extension movements.

The peak torque of internal rotation in the shoulder joint was found to be higher than that of external rotation, and the extension peak torque in the elbow joint was higher than the peak torque of flexion. External/internal rotation rate in the shoulder joint at $240 \%$ velocity was lower compared with the rate at $60 \% \mathrm{~s}$ velocity and also at the rates accepted to be normal for both angular velocities. The elbow flexion/extension rate on the dominant and nondominant sides ranged outside of the rates accepted as normal for both angular velocities.

The present findings can guide the weightlifting athletes to reduce the sports injuries that may occur in shoulder and elbow joints and increase their sports performance.

Key words: Isokinetic, muscle strength, upper extremity, weightlifting

\section{INTRODUCTION}

Performance sports compel the limits of the human body. Increasing the muscle strength for performance improvement and combination of this strength with techniques are the main factors for compelling the limits in the weightlifting field where the technique, explosive power, and flexibility are at the highest level $(1,2)$. The complex structure of weightlifting necessitates examination of muscle strength and the effects of this strength on the body (3). Dynamic stability of the muscles surrounding the upper extremity and shoulder belt is needed for performing a movement successfully in weightlifting (3). A strength imbalance between the muscles that participate in the movement leads to failure in performing the movement successfully and presents a risk factor for upper extremity injuries in athletes. The literature reports that $68.9 \%$ sprain and tendinitis are seen in weightlifters. The regions of injuries in professional weightlifters are the lumbar region (23.1\%), knees (19.1\%), shoulders $(17.7 \%)$, hands (10\%), and elbow $(2.5 \%)(4,5)$. It is known that the agonist/antagonist muscle strength imbalance is among the risk factors in sports injuries (6). Therefore, objective assessment of muscle strength in all athletes is crucial to the sportive success of athletes and prevention of injuries. Despite several studies in the literature exploring the upper extremity isokinetic strength profile in various sports fields such as swimming, handball, volleyball, tennis, water polo, cricket, judo, and basketball (7-22), no study has examined the upper extremity isokinetic muscle strength characteristic in weightlifters. 
The present study was performed to (1) determine the upper extremity isokinetic muscle strength profile of weightlifters and (2) shed light on the exercise and training program by sharing these results with the athletes and team working with them.

\section{MATERIALS AND METHODS}

This study was conducted to determine the characteristics of upper extremity isokinetic muscle strength in weightlifters. It included 21 national Turkish weightlifters, who did not have any orthopedic problems, did professional weightlifting for at least 2 years, were cooperative, had a cognitive state required for the assessment, and volunteered to participate in thestudy. The descriptive characteristics, including age, height, weight, and the dominant-side data of the athletes, were recorded. All the athletes and trainers who agreed to participate in the study were informed about the study purpose, the assessments contained in the study, and the benefits of the study before starting the study. The study had voluntary participation, and was approved by the ethics committee [13/06 (349)].

Isokinetic muscle strength was assessed using the ISOMED $200{ }^{\circledR}$ (Ferstl, Germany) device. The athletes did jogging for 10 min to warm up before starting the tests. Following the warmup, the athletes were taken separately to the isokinetic device to perform the measurements, and the device was adjusted according to their personal anthropometric structures. The weight values were entered into the computer during the test, and the program was set. Whether the movement width of the joint to be tested was appropriate according to the angles to be tested was determined by having the athletes make a sample move at an extremely low speed. At the same time, the gravity effect was set to zero. The joint angles were adjusted in the assessment by considering the joint movement intervals of the persons and the measurement characteristics of the device. The test angles for the shoulder internal rotation (IR)/ external rotation (ER) movement were as follows: An assessment was made in the scapular neutral position between $0^{\circ}$ ER and $90^{\circ}$ $\mathbb{R}$ (the definitions and the protocol used in ISOMED 2000 device) angles for the shoulder flexion/extension movement in the sitting position between $125^{\circ}$ flexion and $10^{\circ}$ extension angles.

All the isokinetic strength measurements were carried out in the sitting position. The shoulder joint IR and ER movements were measured as the arms of the athletes were at $45^{\circ}$ abduction in the scapular plane and the shoulders were at $90^{\circ}$ flexion. This position was selected because the stress on the rotator cuff was minimal in this position compared with the position where the arms were at $90^{\circ}$ abduction in the frontal plane $(12,22)$. On the contrary, previous studies reported that this position was the most reliable position for assessing the shoulder IR and ER strength (14). The elbow joint flexion and extension movements were measured as the volar side of the hand faced the person. This position was selected because it was the position for carrying weights by athletes.

The assessment protocol: The athletes made the IR/ER movement submaximally at $90 \% \mathrm{~s}$ velocity five times, warmed up, and comprehended the movement. Following the warmup movement and a 30-s rest, they made the maximal IR/ER five times at $60 \% \mathrm{~s}$ velocity. Again after a 30-s rest, they made the maximal IR/ER movement 15 times at $240 \%$ s velocity, and the test was completed. The assessments were carried out bilaterally for each joint. First, the dominant side was assessed, and 3 min later, the nondominant side was assessed. The same protocol was applied for the elbow joint the next day. All measurements were performed by an examiner with 3 years of experience during a single test session. The muscle strength was evaluated in terms of peak torque (PT) and average power, calculated from five repeated measurements at an angular speed of $60 \% / \mathrm{s}$ and 15 repeated measurements at an angular speed of 240\%/s. The PT was normalized by body weight (PT/W, percent of torque produced per $\mathrm{kg}$ of BW) to allow comparisons in terms of maximum muscle strength in the shoulder and elbow joints.

The data collected on the isokinetic muscle strength of the athletes were analyzed using Statistical Package for Social Sciences Inc. (IL, USA) for Windows Release 22.0 statistical package program. The descriptive statistics of the variables were determined. The results were expressed as median and standard deviation. Whether the data fitted the normal distribution was examined using the Shapiro-Wilk test, which was defined according to the sample number. Then, the Wilcoxon test was performed for comparing the groups, followed by data analysis.

\section{RESULTS}

The demographic characteristics of the athletes are shown in Table 1.

The PT, PT/W, and the degree and number of repetitions of the PT are shown in Table 2. The shoulder joint IR PT value was found to be higher than that of ER. The PT value for the shoulder ER and IR at $60 \%$ s velocity and for the elbow flexion 
TABLE 1: Basic characteristics of the study participants.

\begin{tabular}{ll}
\hline & $\mathrm{X} \pm \mathrm{SD}$ \\
\hline Age (year) & $16.19 \pm 1.43$ \\
Height $(\mathrm{m})$ & $1.67 \pm 0.06$ \\
Weight $(\mathrm{kg})$ & $65.63 \pm 11.24$ \\
BMI $\left(\mathrm{kg} / \mathrm{m}^{2}\right)$ & $22.76 \pm 3.34$ \\
Sports age (year) & $4.3 \pm 0.8$ \\
\hline
\end{tabular}

and extension movement occurred at the second to third repeat on average, at $240^{\circ} / \mathrm{s}$ velocity for the shoulder $E R$ and $I R$ at the fifth to seventh repeat, and at the fifth to sixth repeat for the elbow flexion and extension. The angle where the PT occurred in the shoulder joint at $60^{\circ} / \mathrm{s}$ velocity was approximately $45^{\circ}-46^{\circ}$ and $66^{\circ}-69^{\circ}$ for the IR and ER, respectively, similar to those on the dominant and nondominant sides $\left(32^{\circ}-33^{\circ}\right.$ and $54^{\circ}-46^{\circ}$, respectively, at $240^{\circ} / \mathrm{s}$ velocity). The angle where the PT occurred for the elbow at $60^{\circ} / \mathrm{s}$ velocity was approximately $77^{\circ}-78^{\circ}$ of flexion and $77-81^{\circ}$ of extension for the flexion and extension, respectively, similar to those on the dominant and nondominant sides. At $240^{\circ} / \mathrm{s}$ velocity, it was $46-48^{\circ}$ of flexion and $91-92^{\circ}$ of extension, respectively, for the flexion and extension movements.

The ratio of the shoulder ER/IR and elbow flexion/extension and comparison of dominant and nondominant sides are shown in Table 3. ER/IR was approximately 47\%-67\% for the dominant side at $60 \%$ in weightlifters, approximately $59 \%-77 \%$ for the nonant side, approximately $37 \%-57 \%$ for the dominant side at $240 \% \mathrm{~s}$ velocity, and approximately 33\%-53\% for the nondominant side. In the present study, the elbow extension PT value was found to be higher than the elbow flexion PT value. The elbow flexion/ extension percentage on the dominant and nondominant sides was determined as $88 \%$ and $83 \%$ at $60 \%$ s velocity and as $77 \%$ and $72 \%$ at $240 \%$ s velocity, respectively. The difference between the right and left sides was found to be more than 10\% at the shoulder IR and elbow flexion at $60 \%$ velocity and the shoulder ER and elbow flexion at $240 \%$ s velocity.

\section{DISCUSSION}

The present study was performed to determine the upper extremity isokinetic muscle strength profile of weightlifters. The PT, PT/W,

TABLE 2: Peak torque, peak torque/weight, and the degree and number of repetitions of peak torque.

\begin{tabular}{|c|c|c|c|c|c|c|}
\hline \multirow{2}{*}{$\begin{array}{l}\text { Shoulder } \\
\text { Test speed }\end{array}$} & \multicolumn{2}{|c|}{ Internal rotation } & \multicolumn{4}{|c|}{ External rotation } \\
\hline & D side & N-D side & $\mathrm{P}^{*}$ & D side & N-D side & $\mathrm{P}^{*}$ \\
\hline $60^{\circ} / \mathrm{s}$ PT $(\mathrm{N} / \mathrm{m})$ & $54.96 \pm 13.88$ & $49.84 \pm 11.57$ & 0.058 & $30.93 \pm 6.47$ & $28.38 \pm 6.26$ & $0.014 *$ \\
\hline $60^{\circ} / \mathrm{s}$ PT/W (N/kg) & $0.79 \pm 0.27$ & $0.76 \pm 0.21$ & 0.203 & $0.51 \pm 0.25$ & $0.46 \pm 0.26$ & $0.002^{*}$ \\
\hline Degree $\left({ }^{\circ}\right)$ & $46.04 \pm 12.63$ & $45.28 \pm 17.77$ & 0.434 & $66.33 \pm 11.99$ & $69.09 \pm 7.32$ & 0.390 \\
\hline Repetitions (piece) & $2.80 \pm 1.28$ & $3.00 \pm 1.44$ & 0.641 & $2.95 \pm 1.46$ & $2.61 \pm 1.32$ & 0.375 \\
\hline $240^{\circ} / \mathrm{s} \mathrm{PT}(\mathrm{N} / \mathrm{m})$ & $50.45 \pm 11.62$ & $48.77 \pm 12.23$ & 0.251 & $23.89 \pm 8.81$ & $21.09 \pm 6.40$ & $0.013^{*}$ \\
\hline $240^{\circ} / \mathrm{s}$ PT/W (N/kg) & $0.74 \pm 0.21$ & $0.73 \pm 0.21$ & 0.543 & $0.36 \pm 0.12$ & $0.31 \pm 0.09$ & $0.003^{*}$ \\
\hline Degree $\left({ }^{\circ}\right)$ & $32.42 \pm 8.77$ & $32.95 \pm 6.52$ & 0.163 & $54.14 \pm 12.94$ & $46.85 \pm 14.13$ & $0.004 *$ \\
\hline Repetitions (piece) & $7.23 \pm 4.04$ & $7.71 \pm 4.16$ & 0.728 & $5.28 \pm 4.67$ & $4.90 \pm 4.19$ & 0.467 \\
\hline Elbow & Flexion & $\mathrm{P}^{*}$ & Extension & $\mathrm{P}^{*}$ & & \\
\hline $60^{\circ} / \mathrm{s}$ PT $(\mathrm{N} / \mathrm{m})$ & $45.40 \pm 12.14$ & $40.90 \pm 8.43$ & $0.005^{*}$ & $49.66 \pm 8.31$ & $50.10 \pm 11.87$ & 0.629 \\
\hline $60^{\circ} / \mathrm{s} \mathrm{PT} \backslash \mathrm{W}(\mathrm{N} / \mathrm{kg})$ & $0.67 \pm 0.21$ & $0.61 \pm 0.16$ & $0.009 *$ & $0.78 \pm 0.25$ & $0.75 \pm 0.25$ & 0.112 \\
\hline Degree $\left({ }^{\circ}\right)$ & $77.80 \pm 18.26$ & $78.38 \pm 22.28$ & 0.673 & $77.23 \pm 10.47$ & $81.38 \pm 14.81$ & 0.271 \\
\hline Repetitions (piece) & $3.33 \pm 2.79$ & $2.71 \pm 2.30$ & 0.619 & $3.19 \pm 2.29$ & $2.95 \pm 1.43$ & 0.840 \\
\hline $240^{\circ} / \mathrm{s}$ PT $(\mathrm{N} / \mathrm{m})$ & $33.50 \pm 9.78$ & $29.76 \pm 8.16$ & $0.033^{*}$ & $43.54 \pm 8.98$ & $41.07 \pm 7.76$ & 0.086 \\
\hline $240 \%$ s PT/W (N/kg) & $0.51 \pm 0.17$ & $0.65 \pm 0.18$ & $0.012^{*}$ & $0.45 \pm 0.15$ & $0.60 \pm 0.15$ & $0.011 *$ \\
\hline Degree $\left({ }^{\circ}\right)$ & $46.52 \pm 11.36$ & $91.61 \pm 11.64$ & 0.340 & $48.19 \pm 11.35$ & $92.52 \pm 9.95$ & 0.924 \\
\hline Repetitions (piece) & $6.14 \pm 4.04$ & $5.66 \pm 3.43$ & 0.601 & $6.80 \pm 4.28$ & $5.38 \pm 5.03$ & 0.844 \\
\hline
\end{tabular}


TABLE 3: Ratio of the shoulder external/internal rotation and elbow flexion/extension and comparison of the dominant and nondominant sides.

\begin{tabular}{|c|c|c|c|c|c|c|}
\hline \multirow[b]{2}{*}{ Joint } & \multirow[b]{2}{*}{ Test speed } & \multicolumn{2}{|c|}{$E R / I R \%$} & \multirow[b]{2}{*}{$\mathrm{P}^{*}$} & \multicolumn{2}{|c|}{ D/N-D side\% } \\
\hline & & D side & N-D side & & IR & $E R$ \\
\hline \multirow[t]{4}{*}{ Shoulder joint } & $60^{\circ} / \mathrm{s}$ & $57.80 \pm 10.75$ & $59.21 \pm 18.29$ & 0.664 & $113.3 \pm 31.2$ & $109.3 \pm 19.4$ \\
\hline & $240^{\circ} / \mathrm{s}$ & $47.10 \pm 9.41$ & $43.82 \pm 9.06$ & 0.099 & $106.18 \pm 16.2$ & $113.36 \pm 19.9$ \\
\hline & & \multicolumn{2}{|c|}{ Flex/ext\% } & & \multicolumn{2}{|c|}{ D/N-D side $\%$} \\
\hline & & D side & N-D side & $\mathrm{P}^{*}$ & Flex & Ext \\
\hline \multirow[t]{2}{*}{ Elbow joint } & $60^{\circ} / \mathrm{s}$ & $88.49 \pm 18.17$ & $83.23 \pm 16.30$ & 0.106 & $111.66 \pm 21.6$ & $105.72 \pm 19.4$ \\
\hline & $240^{\circ} / \mathrm{s}$ & $76.9 \pm 16.26$ & $72.53 \pm 15$ & 0.217 & $115.63 \pm 25$ & $108.45 \pm 19.87$ \\
\hline
\end{tabular}

dominant/nondominant percentage of the athletes at $60 \%$ and $240 \%$ s velocities, percentage of the shoulder IR/ER, and shoulder flexion/extension percentage were assessed, and the upper extremity isokinetic muscle strength profile of the weightlifters was determined.

The shoulder joint IR PT value was found to be higher than the ER PT value in the present study. Considering the studies conducted on the shoulder IR and ER in athletes in the literature, IR PT value was shown to be higher than ER PT value $(7-11,23)$. This outcome was in accordance with the literature and expected in the present study because, considering the muscles surrounding the shoulder, the number of muscles responsible for IR in the shoulder joint was higher than the number of muscles responsible for ER and they were larger in size and stronger (24). Another result of the present study was the repeat number where the PT occurred: athletes achieving the PT value at the 1st repeat and athletes achieving the PT value at the 15th repeat. The results showed that the number of repeats must be increased with the increase in the assessment velocity to reach the PT. Owing to the lack of studies and limited information, it is believed that more extensive large-sample studies need to be conducted on this subject.

No study in the literature was conducted on the angle values where the shoulder ER and IR and elbow flexion and extension PT occurred. This novel study investigated the shoulder ER and IR and elbow flexion and extension PT angle values in weightlifters. It is believed that the integration of the angles where the PT occurred and the movement analysis data of weightlifting sports, and the development of appropriate exercise and training programs, can improve the sportive performances of weightlifters. Furthermore, it is thought that knowing the angles where the PT occurred may guide the establishment of a rehabilitation program in case of a possible injury to the athlete.

In the present study, shoulder ER/IR rotation rate, elbow flexion/ extension rate, and dominant/nondominant rate were estimated as a percentage. These data were crucial because the presence of a balance between the agonist/antagonist muscle strength and the destruction of the balance could make the person prone to injuries (25) Studies by lvey et al and $\mathrm{Ng}$ and Kraemer on normal healthy individuals and recreational athletes demonstrated the ER/R ratio to be $66 \%-75 \%(26,27)$. Alderink and Kuck found this ratio for the shoulder ER/IR to be 66\%-75\% (28). Batalha et al assessed the concentric shoulder ER and IR PT of 60 young swimmers at $60 \%$ and $180^{\circ} / \mathrm{s}$ angular velocities in their study and determined that ER/IR ratio ranged between 70\% and 77\% (9). Saccol et al measured the shoulder ER and IR PT at $60 \%$ and $180 \%$ angular velocities in elite child tennis players and found that this ratio changed between $68 \%$ and $96 \%$ (23). Considering other studies reported in the literature, the ER/IR ratio was reported as $65 \%$ $74 \%$ in cricket players (11), 72\%-74\% in male handball players (15), $78 \%-85 \%$ in professional swimmers $(10), 66 \%-70 \%$ in adolescent handball players (16), 72\%-78\% in women volleyball players (8) $61 \%-64 \%$ in normal healthy male individuals, and $67 \%-69 \%$ in healthy women individuals (7). The present study found that the ER/R was approximately $47 \%-67 \%$ for the dominant side at $60 \%$ in weightlifters, approximately $59 \%-77 \%$ for the nondominant side, approximately $37 \%-57 \%$ for the dominant side at $240 \%$ v velocity, and approximately $33 \%-53 \%$ for the nondominant side. The studies in the literature showed that 
this ratio varied according to the sports fields because each sports field involved specific movements and, therefore, muscle groups developed adaptations unique to the sports. Therefore, the ratio of the agonist/antagonist muscles varied according to healthy individuals because certain muscle groups in different sports fields contracted stronger and faster. It is believed that a lower ER/IR ratio at $240 \%$ in the present study depends on the IR muscles to contain fast-contracting white muscle fibers. The function of fastcontracting muscle fibers is to lift the bar above the head in the movements made during weightlifting. It is necessary to sustain the static stabilization position of the upper extremity muscles and the muscles surrounding the shoulder for keeping the bar above the head. Therefore, the assessment of both strength and velocity parameters at 60 and $240 \%$ s during the isokinetic assessment may be a valuable guidance. It was seen that the assessments were made at 60 and 180\%/s generally. No assessments found that were carried out at $240 \% / s$ on weightlifters. Performing assessments at $240 \% / s$ is crucial for athletes. The present study found that the ER/ IR ratio at $60 \%$ s, which was in accordance with the literature, was lower at $240 \%$ s velocity. A decrease in this ratio was an expected outcome in the assessments carried out at $240 \%$ s velocity because the white muscle fibers were more active. The angular velocities where this ratio existed were as important as the ER/IR ratio during the assessment of the study results. Therefore, it must be considered that the agonist/antagonist ratio can change depending on the fiber types contained by the muscles when the angular velocities change (29). No comparison was made for the findings obtained at $240 \%$ s in the present study because no study available in the literature was conducted on weightlifters. Therefore, this subject needs further investigation.

Keeping in view the studies conducted in the literature, the elbow extension PT values were reported to be higher than the flexion PT values $(17,18)$. In the present study, the elbow extension PT value was found to be higher than the elbow flexion PT value, consistent with the previous findings. However, considering previous studies conducted on the elbow flexion and extension ratio, it was suggested that the flexion/extension ratio must be between 90\% and $100 \%$ at low isokinetic velocities $\left(30^{\circ} \mathrm{s}\right.$ and $\left.60^{\circ} / \mathrm{s}\right)(30,31)$. Knapik and Ramos performed a study on 352 male soldiers. Concentric elbow flexion and extension PT was measured at 30, 90, and $180 \%$ s velocities only on the dominant side, and the flexion/ extension ratio was determined as $114 \%$ and $109 \%$ at 30\%/s and 90\%/s velocity (27). In addition to these studies, Ellenbecker and Mattalino conducted a study on professional baseball players and found the flexion/extension PT ratio to be $103 \%$ and $101 \%$ on the dominant and nondominant sides, respectively (32). The elbow flexion/extension PT ratio on the dominant and nondominant sides at $90 \%$ in elite male child tennis players was found to be $97 \%$ and 108\%, respectively (33). In the present study, the elbow flexion/ extension percentage on the dominant and nondominant sides was evaluated as $88 \%$ and $83 \%$ at $60 \%$ s velocity and between $77 \%$ and $72 \%$ at $240 \%$ s velocity, respectively. The agonist/antagonist muscle strength balance within the elbow joint (in contrast to the shoulder joint) is crucial for preventing sports injuries. It is suggested in the literature that this ratio is close to 1:1. This balance may be disrupted in weightlifting as in many other sports fields due to the nature of the sports. This in turn generates risks for sports injuries. In the present study, this ratio dropped to $72 \%$, in contrast to the studies conducted on other athletes in the literature. This outcome is an indication that extension muscles are stronger than flexor muscles. Considering the injury ratio of the elbow joint shown as 2.5\% in the literature, it is believed that the present study might guide weightlifters and professionals working in this field.

Kannus conducted a study on isokinetic assessments and reported that higher than 10\% difference between the right and left extremities was abnormal (34). The present study found that the difference between the right and left sides was more than 10\% at the shoulder IR and elbow flexion at $60^{\circ} / \mathrm{s}$ velocity and the shoulder ER and elbow flexion at $240 \%$ s velocity. It must be remembered that an imbalance of the muscle strength between the right and left sides can form a basis for scoliosis because the waist and spine injuries and scoliosis are frequently encountered problems due to the nature of weightlifting sports. Therefore, it is thought that symmetrical exercises in the training of weightlifters can diminish the aforementioned possibilities and improve the sportive performance.

The limitation of the present study was that the PT values were not compared based on age and gender because the athletes were few and all were males.

\section{CONCLUSIONS}

Muscle strength is a significant parameter in sportive performance. Isokinetic assessment is an objective method used to measure muscle strength. The present study determined the typical characteristics of upper extremity muscle strength in weightlifters 
using an isokinetic dynamometer. The results revealed the muscular strength characteristics of Turkish professional weightlifting athletes. It is believed that the development of the upper extremity training program in the light of these results can improve the sportive performance and diminish sports injuries.

\section{REFERENCES}

1. Schilling BK, Stone MH, O'bryant HS, Fry AC, Coglıanese $\mathrm{RH}$, Pierce KC. Snatch technique of collegiate national level weightlifters. The Journal of Strength \& Conditioning Research, 2002; 16: 551-555.

2. Souza AL, Shimada SD, Koontz A. Ground reaction forces during the power clean. The Journal of Strength \& Conditioning Research, 2002;16: 423-427.

3. Amca AM, Harbili E, Arıtan S. Koparma kaldırışının biyomekanik analizi için mekanik model geliştirilmesi. Spor Bilimleri Dergisi, 2010; 21: 21-29.

4. Basford JR. Weightlifting, weight training and injuries. Orthopedics, 1985; 8: 1051-1056.

5. Calhoon G, Fry AC. Injury rates and profiles of elite competitive weightlifters. Journal of athletic training, 1999; 34:232.

6. Knapik JJ, Ramos MU. Isokinetic and isometric torque relationships in the human body. Archives of physical medicine and rehabilitation, 1980; 61: 64-67.

7. Shklar A, Dvir Z. Isokinetic strength relationships in shoulder muscles. Clinical biomechanics, 1995;10: 369-373.

8. Alfredson H, Pietilä T, Lorentzon R. Concentric and eccentric shoulder and elbow muscle strength in female volleyball players and non-active females. Scandinavian journal of medicine \& science in sports, 1998; 8: 265-270.

9. Batalha NMP, Raimundo AMDM, Tomas-Carus P, Fernandes ODJSM, Marinho DA, Silva AJRMD. Shoulder rotator isokinetic strength profile in young swimmers. Revista Brasileira de Cineantropometria \& Desempenho Humano, 2012; 14: 545-553.

10. West D, Sole G, Sullivan SJ. Shoulder external and internal rotation isokinetic strength in master's swimmers. J Sport Rehabil, 2005; 14: 12-19.

11. Mabasa XM, Stewart A, Fleishman C. Isokinetic strength of shoulder internal and external rotators in cricket bowlers. South African Journal of Physiotherapy, 2002; 58: 35-39.

12. Greenfield $B$, Donatelli $R$, Wooden $M$, Wilkes J. Isokinetic evaluation of shoulder rotational strength betweenthe plane of scapula and the frontal plane. The American Journalof Sports Medicine, 1990; 18: 124-128.

13. Kuhlman J, Iannotti J, Kelly M, Riegler F, Gevaert M, Ergin TM. Isokinetic and isometric measurementof strength of external rotation and abduction of the shoulder.The Journal of Bone and Joint Surgery, 1992; 74: 1320-1333.

14. Edouard P, Samozino P, Julia M, Gleizes Cervera S, Vanbiervliet W, Calmels P, Gremeaux V. Reliability of isokinetic assessment of shoulder-rotator strength: a systematic review of the effect of position. J Sport Rehabil, 2011; 20: 367-383.

15. Andrade MS, Vancini RL, de Lira CA, Mascarin NC, Fachina RJ, Silva ACD. Shoulder isokinetic profile of male handball players of the Brazilian National Team. Brazilian journal of physical therapy, 2013; 17: 572-578.
16. Pontaga I, Zidens J. Shoulder Rotator Muscle Dynamometry Characteristics: Side Asymmetry and Correlations with BallThrowing Speed in Adolescent Handball Players. Journal of human kinetics, 2014; 42: 41-50.

17. Ruivo R, Pezarat-Correia P, Carita AI. Elbow and shoulder muscles strength profile in judo athletes. Isokinetics and Exercise Science, 2012; 20: 41-45.

18. Ellenbecker TS, Roetert EP. Isokinetic profile of elbow flexion and extension strength in elite junior tennis players. Journal of Orthopaedic \& Sports Physical Therapy, 2003; 33: 79-84.

19. Lategan L, Kruger PE. Effect of two different handgrip positions on elbow peak torque values. 2007.

20. Harput G, Guney H, Colakoglu FF, Baltacl G. Isokinetic Strength Profile of Shoulder Internal and External Rotators of Adolescent Volleyball Players. Orthopaedic Journal of Sports Medicine, 2014; 2

21. dos Santos Andrade $M$, de Lira $C A B$, Vancini $R L$, de Almeida $A A$, Benedito-Silv, AA, da Silva AC. Profiling the isokinetic shoulder rotator muscle strength in 13-to 36-year-old male and female handball players.Physical Therapy in Sport, 2013;14: 246-252.

22. Wang HK, Macfarlane A, Cochrane T. Isokinetic performance and shoulder mobility in elite volleyball athletes from the United Kingdom. British Journal of Sports Medicine, 2000; 34: 39-43.

23. Saccol MF, Silva RT, Gracitelli G, Laurino CFS, Andrade MS. Concentric and eccentric isoknetic strength profile of shoulder rotators in elite junior tennis players. In XXV ISBS Symposium, Ouro Preto-Brazil, 2007.

24. Dark A, Ginn KA, Halaki M. Shoulder muscle recruitment patterns during commonly used rotator cuff exercises: an electromyographic study. Physical therapy, 2007; 87: 10391046.

25. Knapik JJ, Bauman $\mathrm{CL}$, Jones $\mathrm{BH}$, Harris JM, Vaughan L. Preseason strength and flexibility imbalances associated with athletic injuries in female collegiate athletes. The American Journal of Sports Medicine, 1991; 19: 76-81.

26. Ivey FM, Calhoun JH, Rusche K, Bierschenk J. Isokinetic testing of shoulder strength:normal values. Archives of Physical Medicine and Rehabilitation, 1985; 66: 384-6.

27. $\mathrm{Ng} \mathrm{LR}$, Kraemer JS. Shoulder rotator torques in female tennis and nontennis players..Journal of Orthophedic Sports Physical Therapy, 1991;13:40-7.

28. Alderink G, Kuck D. Isokinetic shoulder strength of high school and college-aged pitchers. Journal of OrthopedicsSports Phyisiotherapy Therapie, 1986; 7: 163-172.

29. Srinivasan RC, Lungren MP, Langenderfer JE, Hughes RE. Fiber type composition and maximum shortening velocity of muscles crossing the human shoulder. Clinical anatomy, 2007;20: 144-149.

30. Perrin $\mathrm{DH}$. Isokinetic exercise and assessment. Human Kinetics. 1993.

31. Heyward VH, Gibson A. Advanced fitness assessment and exercise prescription 7th edition. Human kinetics, 2014.

32. Ellenbecker TS, Mattalino AJ. The elbow in sport. Champaign IL: Human Kinetics Publishers. 1997.

33. Ellenbecker TS, Roetert EP. Isokinetic testing and training in tennis. Isokinetics in human performance, 2000; 358-377. 